\title{
The Effects of Magnetic Fields on the Winds from Luminous Hot Stars
}

\author{
Joseph P. Cassinelli and Nathan A. Miller \\ University of Wisconsin, Astronomy Department, 475 N. Charter St. Madison WI. \\ 53706, USA
}

\begin{abstract}
Here we explore three types of models in which the combination of magnetic fields with line driving forces leads to faster winds than line forces alone. The fields can change the flow geometry; they can couple with rotation and transmit angular momentum; and they can carry transverse Alfvén waves. All three can lead to a deposition of momentum beyond the critical point and accelerate winds. Some history of the attempts to use magnetic fields to understand hot star winds is discussed and several new models for magnetic rotator $\mathrm{B}[\mathrm{e}]$ winds are presented.
\end{abstract}

\section{Introduction}

There are several lines of evidence that magnetic fields play a role in hot star winds. The co-rotating interaction region (CIR) models of Cranmer and Owocki (1996) require a longitudinal dependence of the wind properties, which can arise naturally in stars with significant magnetic fields. There are correlations between $\mathrm{H} \alpha$ and $\mathrm{He}$ II $4686 \AA$ that form at the base of winds and the wings of UV line profiles that form far from the star (Kaper et al. 1996). The X-rays from OB stars show anomalies that require a multi-component wind structure (Cohen et al. 1997). All of these can be taken as evidence that fields near the star affect conditions far out in the wind. In this paper, we will focus on just one question: How can a combination of magnetic wind forces and line driven wind forces lead to faster outflows than would arise from line forces alone? We review three basic effects of fields: a geometrical effect, a rotational effect, and a transverse Alfvén wave effect.

First, let us briefly summarize the history of magnetic modelling of the winds of luminous hot stars. In 1979, it became clear for the first time that Wolf-Rayet stars have a "wind momentum problem", i.e. the ratio of the wind momentum to the radiative momentum, $\eta=\dot{M} v_{\infty} /\left(L_{*} / c\right)$, is much larger than unity- by factors as high as $\eta=30$ to 50 . Currently it is widely thought that the problem can be explained by multiple scattering of radiation (Gayley et al. 1995), but when first discovered the momentum ratio seemed to pose an insurmountable problem for radiation driven wind theory. Because of this difficulty with radiation driven wind theory Hartmann and Cassinelli (1981) and Cassinelli (1982) investigated whether magnetic forces could explain the winds. Since the momentum excess in the wind was so much greater than the radiation field, the effects of radiation were ignored and the focus was on the 
effects of fields alone. Both magnetic rotator models and Alfvén wave driven wind models were found capable of driving the wind of a WR star having $\dot{M}=2 \times 10^{-5} M_{\odot} \mathrm{yr}^{-1}$ and $v_{\infty} \approx 1800 \mathrm{~km} \mathrm{~s}^{-1}$.

The rotating models were based on the fast magnetic rotator theory of Hartmann and MacGregor (1980). To drive the WR wind it required the star to have a field of $20,000 \mathrm{G}$ and be rotating at $95 \%$ critical, both a field and a rotation rate which seemed implausible.

The Alfvén wave driven wind theory developed by Hartmann and MacGregor (1982) required the same large field, a wave flux of $1.1 \times 10^{14} \mathrm{ergs}$ $\mathrm{cm}^{-2} \mathrm{sec}^{-1}$, assuming a damping length of $1 R_{*}$. In this model both the field and the wave luminosity $\left(=1 / 3 L_{*}\right)$ seemed excessive.

During the subsequent decade, Maheswaran and Cassinelli $(1988,1992)$ developed upper and lower limits on surface fields based on virial theorem considerations, and the submersion of surface fields by the effects of circulation currents. Models combining radiation and magnetic forces were also developed and these will be discussed below. Missing throughout the history of the subject have been observational detections of magnetic fields. This appears to be the area that should show the greatest improvement in the future.

\section{Flow Tube Geometrical Effects}

MacGregor (1988) investigated the effects of non-spherical expansion on radiation driven winds. Seemann (1998) has also investigated the effects of flow tube geometry for luminous magnetic rotators. In the geometrical model of MacGregor, there is a velocity change that arises from a modification of the conservation of mass equation. Normally we use $\rho(r) v(r) r^{2}=$ constant to eliminate the density from the wind momentum equation. However, for the case of a non-radial outflow, MacGregor uses $\rho(r) v(r) r^{2} \mathbf{f}(r)=$ constant, where $f(r)$ is illustrated in Figure 1. The factor arises in the case of the solar wind in coronal hole regions, where the base of the tube is constricted by neighboring closed field regions.

The factor $f(r)$ appears in the wind momentum equation both in the gas pressure gradient $\rho^{-1} d p / d r$ and in the CAK line driven wind acceleration term $\left(\rho^{-1} d v(r) / d r\right)^{\alpha}$.

The results of the non-radial divergence factor are as follows:

a) the flow tube geometry can lead to an increase of the flow speed by a factor of two to three relative to the pure CAK wind case, for example increasing the wind speed from $1500 \mathrm{~km} \mathrm{~s}^{-1}$ to $3000 \mathrm{~km} \mathrm{~s}^{-1}$. This increase appears to be more than enough to account for the most extreme velocity differences needed to explain discrete absorption components (DAC's) as proposed in the CIR models.

b) the mass loss rate remained unchanged from the CAK result in MacGregor's models. This is explained by the fact that although the flow tubes mod- 


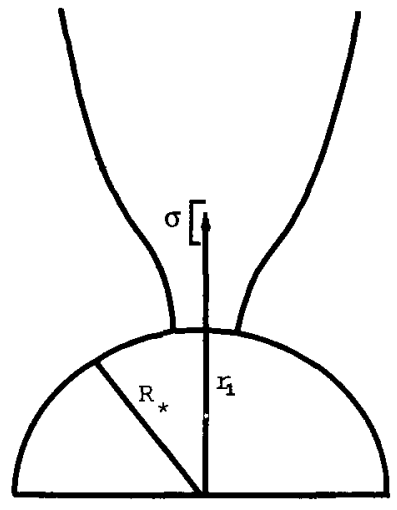

Fig. 1. The non-radial flow tube geometry used by MacGregor (1988). This particular case led to an enhancement of the wind speed by a factor of three.

ified the location of the CAK critical point, the actual velocity distribution in the subcritical point region was not changed significantly. In other words, the flow tube effect is an example of the "afterburner" effect (Cassinelli and Castor, 1973, Leer and Holzer, 1978). This states that momentum changes concentrated in the region beyond the critical point affect the terminal velocity but not the mass loss rate. If the momentum is also deposited in the subcritical region it will affect the mass loss rate as well. (see $\mathrm{Ch} 3$, Lamers and Cassinelli, 1998)

\section{Luminous Magnetic Rotator Theory}

The basic physics explaining how an open magnetic field emerging from a rotating star will affect the mass flux, and the radial and azimuthal components of velocity was developed for the solar wind by Weber and Davis (1967). The equations describing how radiation forces can drive a flow from a luminous star were developed by Castor, Abbott, and Klein (1975, CAK). Luminous Magnetic Rotator (LMR) theory concerns the combination of the forces treated in these two papers. Cassinelli (1998) has recently reviewed the development of the theory in a sequence of papers by Friend and MacGregor (1984), Poe and Friend (1986), and Poe, Friend, and Cassinelli (1989).

There are three domains of magnetic rotator theory. A star can be a slow magnetic rotator (SMR), a fast magnetic rotator (FMR), or a centrifugal magnetic rotator (CMR). The defining characteristic of an SMR wind is that the magnetic field and rotation have almost no effect on the values of either the mass loss rate or wind terminal velocity. So, for an SMR, the values for $\dot{M}$ and $v_{\infty}$ are completely set by the primary mechanism. However, even for an SMR wind there is a transfer of angular momentum from the star to the wind, which leads to a spin down of the star. 
In the case of a FMR wind, the deposition of the momentum occurs mostly in the region beyond the inner critical point, and thus the terminal velocity is increased, but with little change in the mass loss rate.

Centrifugal magnetic rotator theory was developed by Hartmann and MacGregor (1982), regarding mass loss from rapidly rotating protostars. The magnetic field causes solid body rotation out to the inner critical point. Thus, there is a simple modification owing to centrifugal forces to the hydrostatic density distribution, and this causes $\dot{M}$ to increase. The terminal velocity of a centrifugal magnetic rotator is given to a very good approximation by the speed at the fast critical point, $v_{M}$, called the Michel velocity

$$
v_{M}{ }^{3}=\left(R_{*}{ }^{4} \Omega^{2} B_{r, 0}{ }^{2} / \dot{M}\right) \approx v_{\infty}{ }^{3} .
$$

Note here that the terminal velocity is fully determined by the basic parameters at the base of the wind, the rotation rate, $\Omega$ (which determines the mass loss rate $\dot{M}$ ), and the magnetic field at the surface of the star. So unlike $\dot{M}$, the value of $v_{\infty}$ depends explicitly on the field strength; given a rotation rate, the higher the field the faster the wind.

Radiation also plays a role in increasing the velocity of the wind along the FMR portion of the track in the $\dot{M}$ versus $v_{\infty}$ plot. Because of the velocity gradient effect, the radiative acceleration is enhanced by the effects of the strong magnetic field. There is an increase the radiation force because in Sobolev line transfer theory the larger the velocity gradient the larger the amount of stellar radiation that is Doppler shifted into the line absorbing region per unit length in the wind. Thus, the magnetic rotator forces make the stellar radiation field more effective, per spectral line, in driving a wind.

These effects are illustrated in figures 2 and 3 for the case of a B[e] star (as originally treated by Cassinelli et al. 1989). We compare a non-rotating star with one rotating at $60 \%$ critical and with one that is rotating at that rate but which also has a magnetic field of $500 \mathrm{G}$. Though radiation remains the dominant force throughout the wind, the extra acceleration caused by the magnetic force desaturates the radiation force, making it more effective at driving much faster winds than would be achieved otherwise.

\section{Alfvén Wave Amplified Winds from Hot Luminous Stars}

There are many similarities between Alfvén wave driven winds and magnetic rotator winds. In the case of the magnetic rotators centrifugal forces and the Lorentz force associated with the curvature of the field lines transmit momentum to the wind. In the Alfvén case the origin of the force is presence of an oscillation at the base of the field lines, this then leads to the deposition of momentum both by a centrifugal force and a Lorentz force as the wave disturbance propagates through the plasma. The basic process as applied to 


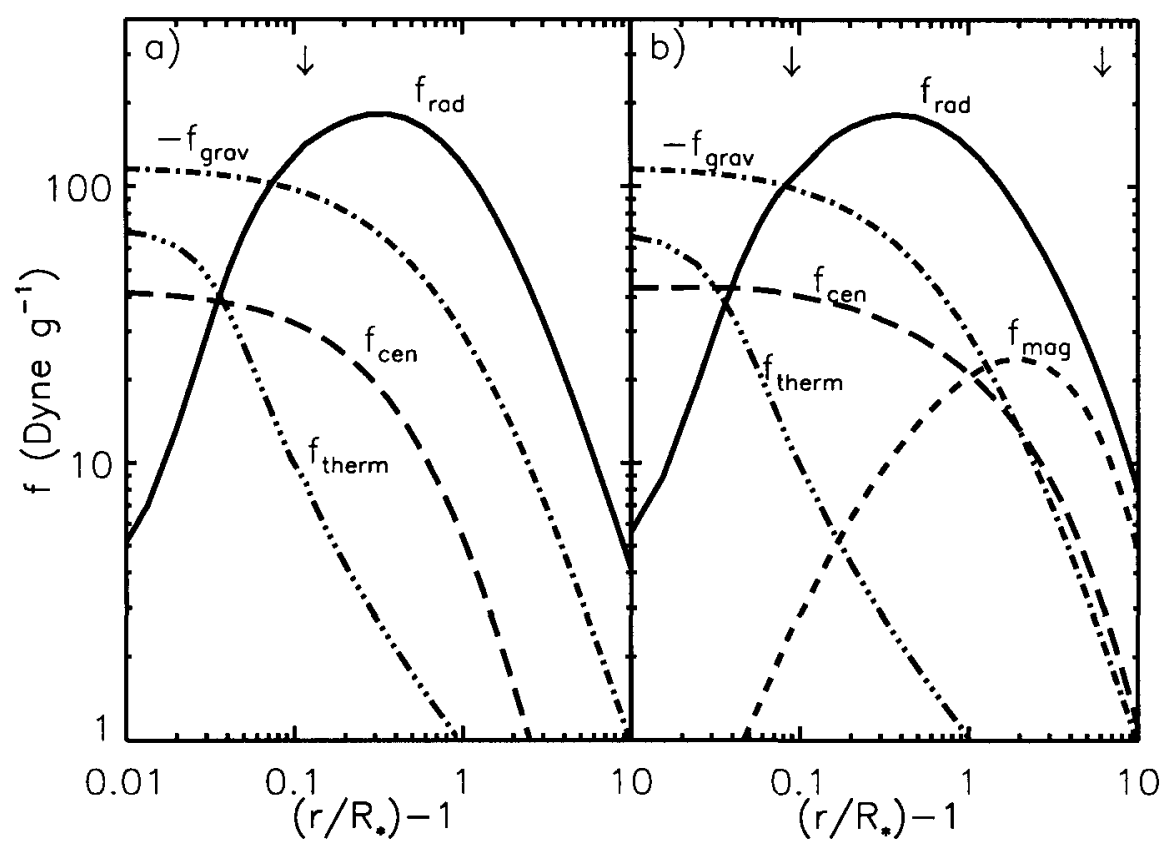

Fig. 2. The distribution, as a function of radius, of 5 forces per unit mass: thermal, magnetic, centrifugal, gravitational, and line radiation, for a $\mathrm{B}[\mathrm{e}]$ star which is assumed to rotate at $60 \%$ critical speed. Panel (a) shows results for a model star with no magnetic field, resulting in a terminal velocity of $1200 \mathrm{~km} \mathrm{~s}^{-1}$, and a mass-loss rate of $6.6 \times 10^{-6} M_{\odot} \mathrm{yr}^{-1}$. The arrow indicates the inner critical point. For Panel (b), on the right, $B_{r, o}=500 \mathrm{G}$, and we see the added magnetic force leads to a steeper velocity gradient near the Alfvén radius resulting in a visibly increased radiation force. Both the inner and Alfvén critical points are indicated. This highly magnetic model has a terminal velocity of $2000 \mathrm{~km} \mathrm{~s}^{-1}$, much faster than the panel (a) model, and a mass loss rate $\left(7.9 \times 10^{-6} M_{\odot} \mathrm{yr}^{-1}\right)$ that is only slightly larger than the panel (a) model, as is characteristic of models in the FMR regime (from Friend and MacGregor, 1984).

coronal winds is described by Holzer, Flå, and Leer (1983). In the case of the sun, Alfvén waves are considered responsible for driving the fast $(\approx 800$ $\mathrm{km} \mathrm{s}^{-1}$ ) outflows from coronal hole regions. The mechanism was proposed for explaining the outflows of red giants by Hartmann and MacGregor (1982). However Holzer et al. (1983) criticized the model because it depended too sensitively on the wave damping length. This is because the winds of red giants are slow, while the forces tend to lead to fast winds. In the case of hot stars the flow speed produced by the Alfvén waves is not a problem because the winds tend to be fast in any case, and we are using the waves to produce a 


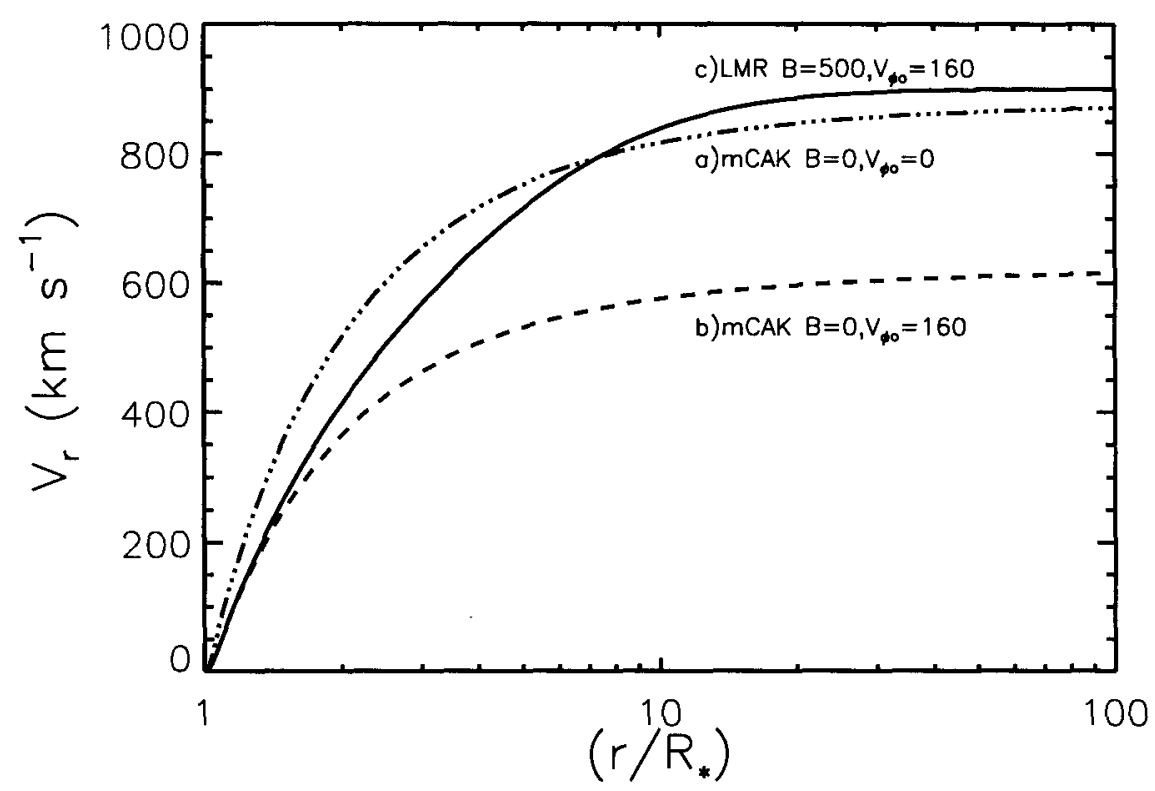

Fig. 3. The radial velocity distribution for three $B[e]$ models: Curve a) shows the velocity distribution for a mCAK (Friend and Abbott (FA) 1986) model for a star with no rotation or magnetic field. Curve b) is for a FA model that includes rotation. Note this reduces both the velocity near the star and the terminal velocity (which scales as $v_{\text {esc }}$ ). Curve $\mathrm{c}$ ) is for a rotating magnetic model. Near the star the model is similar to the purely rotating model, but farther out near the Alfvén point the wind is accelerated by magnetic force effects.

wind that is even faster than can be produced by radiation alone. The Alfvén wave forces have been combined with radiation forces by Dos Santos, JatencoPereira, and Opher (1993), specifically to explain the Wolf-Rayet momentum problem that was discussed in the introduction. Dos Santos et al. considered WR outflows with Alfvén waves and the modified CAK forces. Unfortunately they also included the effects of the non-radial spherical outflow discussed in section 2, so it is not possible to isolate the Alfvén wave effects. The Alfvén models lead to the following conclusions:

a) The mass loss rate is determined by the average of the square of the transverse wave amplitude $\left\langle\left(\delta V_{\phi}\right)^{2}\right\rangle$ This is analogous to the centrifugal magnetic rotator case in which $\dot{M}$ is determined by the (transverse) rotation velocity at the base of the magnetic tubes.

b) The terminal velocity is determined by the magnitude of the B-field, as 
most of the deposition of momentum occurs beyond the inner critical point, but near the Alfvén radius. This again is analogous to the CMR case in which the magnetic field determines $v_{\infty}$.

c) Dos Santos et al. could achieve WR wind conditions with a magnetic field of less than $1000 \mathrm{G}$. The model had sufficient momentum to achieve a momentum ratio of $\eta \approx 10$. Furthermore the model had an energy flux of less than about 1 percent of the radiative luminosity.

The combined Alfvén wave plus line driven wind model has not been pursued recently perhaps because: a) It is not clear what would produce the oscillations at the base of the winds. b) Transverse motions in general may be strongly damped by the interaction with the radiation forces (Owocki and Rybicki, 1986). Whether this is a problem or not is unclear as an unknown damping force is incorporated in the equations in any case. c) It is also not clear whether the Dos Santos et al. solution crossed mCAK type critical points, and this is a major problem and concern of any model that uses line driving forces.

\section{$5 \quad$ Summary}

Our goal has been to investigate whether the combination of radiation and stellar magnetic fields could increase the terminal velocity of the wind from a hot luminous star. We have shown that there are three ways that this can be done: 1) a non-spherical divergence of area in flow tubes defined by open field geometries, 2) fast magnetic rotator forces 3) Alfvén wave forces, at least in combination with non-spherical geometries. We have indirect evidence for the presence of fields as given by fast wind slow wind collision effects and the dependence of wind properties on the rotation of the star. What is most seriously needed is direct observational information about field strengths and field geometries. Several of us have been studying the Hanle effect (Cassinelli and Ignace 1996, Ignace Nordsieck and Cassinelli, 1997,1998). This effect requires high-resolution polarimetric observations in the far-ultraviolet, and only a rocket experiment has been approved thus far. There is no doubt that magnetic fields are capable of producing the general fast wind slow wind phenomena or rotational modulations that are inferred from observations. The problem is that there remain too few observational/diagnostic constraints to guide more detailed theoretical modelling. Here we have focussed on changes that fields can induce regarding the terminal velocity. In principle the fields can also lead to a mass-loss rate that varies across the face of a hot star. Perhaps it is possible to distinguish between the two effects, and this will provide a better understanding between forces acting primarily at the base of the wind and those operating beyond the wind critical point. 


\section{References}

Castor, J.I., Abbott, D.C., and Klein, R.I. 1975, ApJ, 195, 157. (CAK)

Cassinelli, J.P., Schulte-Ladbeck, R.E., Abbott, M., and Poe, C.H. 1989, in Proc. IAU Colloq. 113, Physics of Luminous Blue Variables, Moffat, A.F.J., Lamers, H.G.J., eds., Dordrecht, Kluwer, p. 121.

Cassinelli, J.P. 1998, to appear in ESO workshop, Cyclical Variability in Stellar Winds.

Cassinelli, J.P., and Castor, J.I. 1973, ApJ, 179, 189.

Cassinelli, J.P. and Ignace, R., 1996, in Proc. 33rd Liege Intl. Astrophys. Colloq., Wolf-Rayet Stars in the Framework of Stellar Evolution, Vreux, J.M., Detal, A., Fraipont-Caro, D., Gosset, E., Rauw, G., eds., p. 531.

Cohen, D.H., Cassinelli, J.P., MacFarlane, J.J. 1997, ApJ, 487,876.

Cranmer, S.R. and Owocki, S.P. 1996, ApJ, 462, 469.

Dos Santos, L.C., Jatenco-Pereira, V., and Opher, R. 1993, ApJ, 410, 732.

Friend, D.B., and Abbott, D.C. 1986, ApJ, 311, 701.

Friend, D.B., and MacGregor, K.B. 1984, ApJ, 282, 591.

Gayley, K.G., Owocki, S.P., and Cranmer, S.R. 1995, ApJ, 442, 296.

Hartmann, L., and Cassinelli, J.P. 1981, BAAS, 13, 795.

Hartmann, L., and MacGregor, K.B. 1980, ApJ, 242, 260.

Hartmann, L., and MacGregor, K.B. 1982, ApJ, 257, 264.

Holzer, T.H., Flå, T., and Leer, E. 1983, ApJ, 275, 808.

Kaper, L., Henrichs, H.F., Nichols, J.S., Snoek, L.C., Volten, H., and Zwarthoed, G.A.A. 1996, A\&AS 116,257.

Ignace, R., Nordsieck, K.H., and Cassinelli, J.P. 1997, ApJ, 486, 550.

Ignace, R., Nordsieck, K.H., and Cassinelli, J.P. 1998, ApJ, submitted.

Lamers, H. J. G. L. M. and Cassinelli, J. P. 1998: Introduction to Stellar Winds, (Cambridge, Cambridge), Chap. 9.

Leer, E., Holzer, T.E. 1979, Solar Phys., 63, 143.

MacGregor, K.B. 1988 ApJ, 327, 794.

Maheswaran, M., and Cassinelli, J.P. 1988 ApJ, 335, 931.

Maheswaran, M., and Cassinelli, J.P. 1992 ApJ, 386, 695.

Owocki, S.P., and Rybicki, G.B. 1986, ApJ, 309, 1270.

Poe, C.H., and Friend, D.B. 1986, ApJ, 311, 317.

Poe, C.H., Friend, D.B., and Cassinelli, J.P. 1989, ApJ, 337, 888.

Seeman, H. 1998, Thesis, University of Bonn.

Weber, E. J., and Davis, L. Jr. 1967, ApJ, 148, 217.

\section{Discussion}

S. Owocki: In a paper with George Rybicki in 1985, we showed that horizontal velocity perturbations in a line-driven wind will be strongly damped by the line-drag effect. This suggests that radially propagating Alfvén waves, with horizontal velocity fluctuations, should be strongly damped.

J. Cassinelli: Thanks for reminding me about that. It would be good to consider specifically the case of transverse Alfvén waves. 
H. Henrichs: Can you make a quantitative estimate of the magnetic field needed to provide the link between $\mathrm{H} \alpha$ and the UV fast wind?

J. Cassinelli: Sure, if you can tell me the velocity contrast needed to provide the observed DAC. That velocity difference can be used in conjunction with the $\dot{M}$ versus $v_{\infty}$ plot of Luminous Magnetic Rotator theory. The larger the $B$ field, the greater the difference in velocity between a fast magnetic rotator and the mCAK velocity associated with a non-magnetic longitude sector.

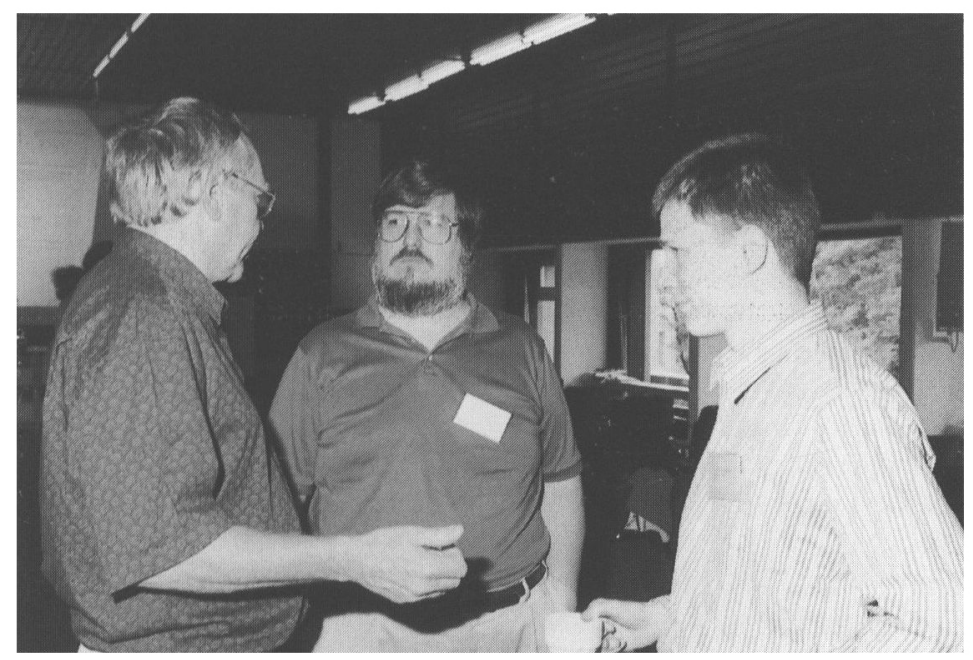

Joseph Cassinelli, Jon Bjorkman and Richard Ignace 